\title{
PROGRESS REPORT ON 21-GM. RESEARGH BY THE NETHERLANDS FOUNDATION FOR RADIO ASTRONOMY AND THE LEIDEN OBSERVATORY
}

\author{
G. WESTERHOUT \\ University Observatory, Leiden, Netherlands
}

From November I953 to August 1955, about 2500 line profiles have been measured with the Kootwijk receiver, under the supervision of Ir C. A. Muller. His receiver is of the well-known frequency-switching type. Two pairs of channels in the second i.f. amplifier, about $5 \mathrm{Mc}$./s., have bandwidths of $36 \mathrm{kc}$./s. The components of one pair are $1080 \mathrm{kc} . / \mathrm{s}$. apart; the second pair is shifted $500 \mathrm{kc}$./s. with respect to the first. Each pair of channels, combined with the continuously variable second local oscillator and with the fixed, crystal-controlled pair of first local oscillators, which are also $1080 \mathrm{kc} . / \mathrm{s}$. apart, gives a switching system. The power at a certain frequency is compared with that at a $1080 \mathrm{kc}$./s. higher frequency during half the switching period and with that at a $1080 \mathrm{kc}$./s. lower frequency during the other half by switching between the two first local oscillators. The signals are fed through an integrating network with a time constant of $54 \mathrm{sec}$. At the outputs of the two pairs are two separate recorders, each giving one-half (or more) of a line profile (see Fig. I). A continuous frequency calibration of the second local oscillator provides $10 \mathrm{kc}$./s. markers on the records, accurate to $I$ in $10^{6}$. The limiting sensitivity is $0.7^{\circ} \mathrm{K}$., with an overall noise figure of $6.3(8 \cdot 0 \mathrm{db}$.). The $7.5 \mathrm{~m}$. Würzburg aerial has a beam-width between half-power points of $1: 9 \times 2: 7$.

The programme, finished during a nearly continuous one-and-a-half years operation, was mainly concentrated on a study of the large-scale structure of the galactic system. It consisted of the investigation of a strip around the galactic equator, where line profiles were obtained, and special studies based on these profiles were made, as summarized in points (I) to (6) below. Some additional investigations are mentioned in points (7) to (10).

(I) Line profiles from $l=320^{\circ}$ to $l=42^{\circ} \cdot 5$, spaced $2{ }^{\circ} 5$ in longitude, at latitude $-1: 5$ and corresponding profiles at latitudes of approximately 
$-5: 5,-3: 5,+0 \bullet^{\circ},+2 \bullet_{5}$ and $+4{ }^{\circ} 5$ along the same declination circles. A study of these profiles, combined with the measures mentioned in point (4), is reported in the contribution by M. Schmidt to this Symposium (paper 6).

(2) Line profiles from $l=45^{\circ}$ to $l=110^{\circ}, b=+10^{\circ}$ to $-10^{\circ}$, spaced $2^{\circ} \cdot 5$ in longitude and 2.5 in latitude.

(3) Line profiles from $l=115^{\circ}$ to $l=220^{\circ}, b=+10^{\circ}$ to $-10^{\circ}$, spaced $5^{\circ}$ in longitude and $2{ }^{\circ}$ in latitude. At selected points the latitude interval was somewhat extended. The results of the study of the profiles in points

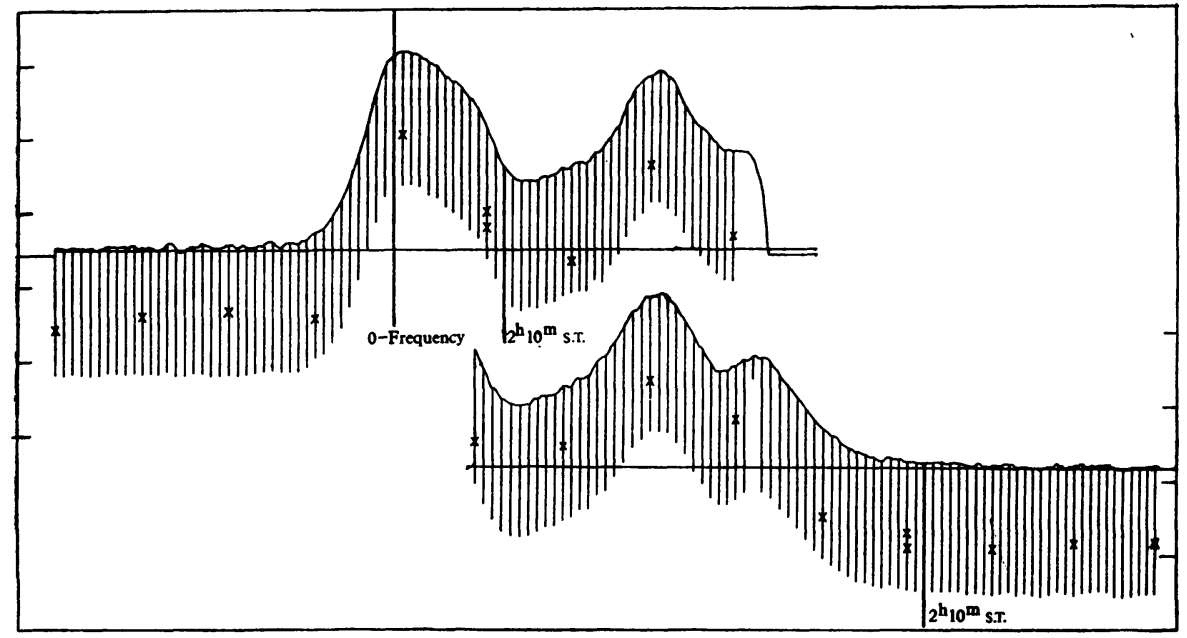

Fig. I. A double line profile at $l=67^{\circ}, b=+2{ }_{5}^{\circ}$, as recorded simultaneously on two recorders. The vertical lines are $10 \mathrm{kc}$./s. frequency markers. The temperature of the main top is $64^{\circ} \mathrm{K}$.

(2) and (3) are to be found in the next paper. Fig. 2 shows the line profiles at or near the galactic equator that are contained in programmes $\mathrm{I}-3$.

(4) About 200 drift-curves were obtained by M. Schmidt, at fixed frequency and position, in the longitude range $l=340^{\circ}$ to $35^{\circ}$. These served to study the latitude distribution in the central regions.

(5) The positive velocity ends of the line profiles in the region $l=320^{\circ}$ to $l=40^{\circ}$ were studied by K. K. Kwee, C. A. Muller and G. Westerhout[1]. The circular velocity as a function of the distance from the galactic centre was determined with a hitherto unattained accuracy. Long wings of very low intensity, extending to radial velocities of $200 \mathrm{~km}$. $/ \mathrm{sec}$., were discovered close to the centre and are discussed in the same paper. They justify the hypothesis that the hydrogen in the region $R<2.5$ kiloparsecs is in a highly turbulent state. 
(6) M. Schmidt made a study of the regions where the optical thickness of the neutral hydrogen may be assumed to be very high: $(a)$ the centre, where radial velocity due to galactic rotation is zero and the density is high; and (b) galactic longitudes $l=40^{\circ}-45^{\circ}$, where the line of sight is almost tangent to a circle through the sun, around the centre, and passes through the Orion spiral arm over a considerable distance. A new value of the antenna temperature at infinite optical thickness, $T_{0}$, was obtained by means of the formula $T=T_{0}\left(\mathrm{I}-e^{-\tau}\right)$. After correction for aerial pattern, a minimum value $T_{0}=125^{\circ} \mathrm{K}$. was found for both regions. Although it is realized that local variations in the temperature are quite likely, a constant $T_{0}=125^{\circ} \mathrm{K}$. was used throughout all the reductions.

It should be noted that the absolute value of this temperature is based on the noise figure quoted. This noise figure in turn was determined by assuming that the noise increase when the aerial is pointed into a nearby pine-wood corresponds to $280^{\circ} \mathrm{K}$. It is felt that this value is probably correct to within Io $\%$. The intensity scale was checked daily by measuring the main top of the profile at $l=50^{\circ}, b=0^{\circ}$, the height of which corresponds to $100^{\circ} \mathrm{K}$.

(7) A study of the Taurus dark regions was made by Dr F. D. Kahn, to obtain information about the relation between dust and interstellar gas. The preliminary results based on four selected points and published in $B . A . N$. no. $45^{2[2]}$ indicate that the regions of very strong obscuration
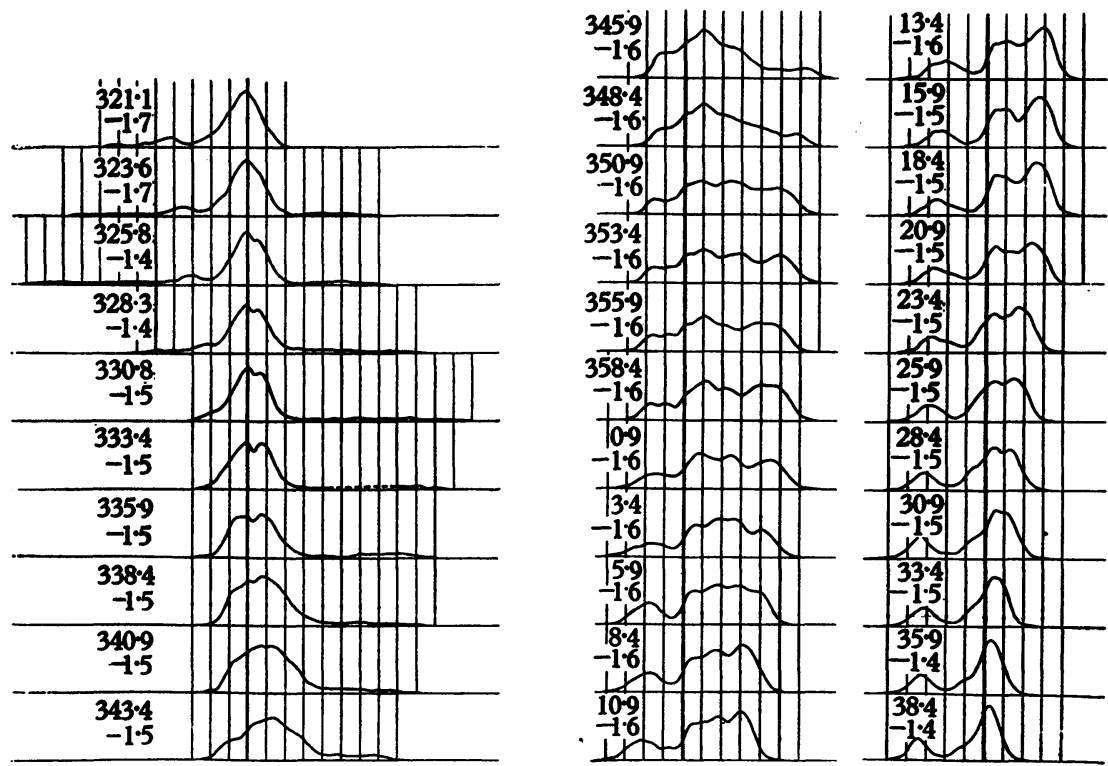


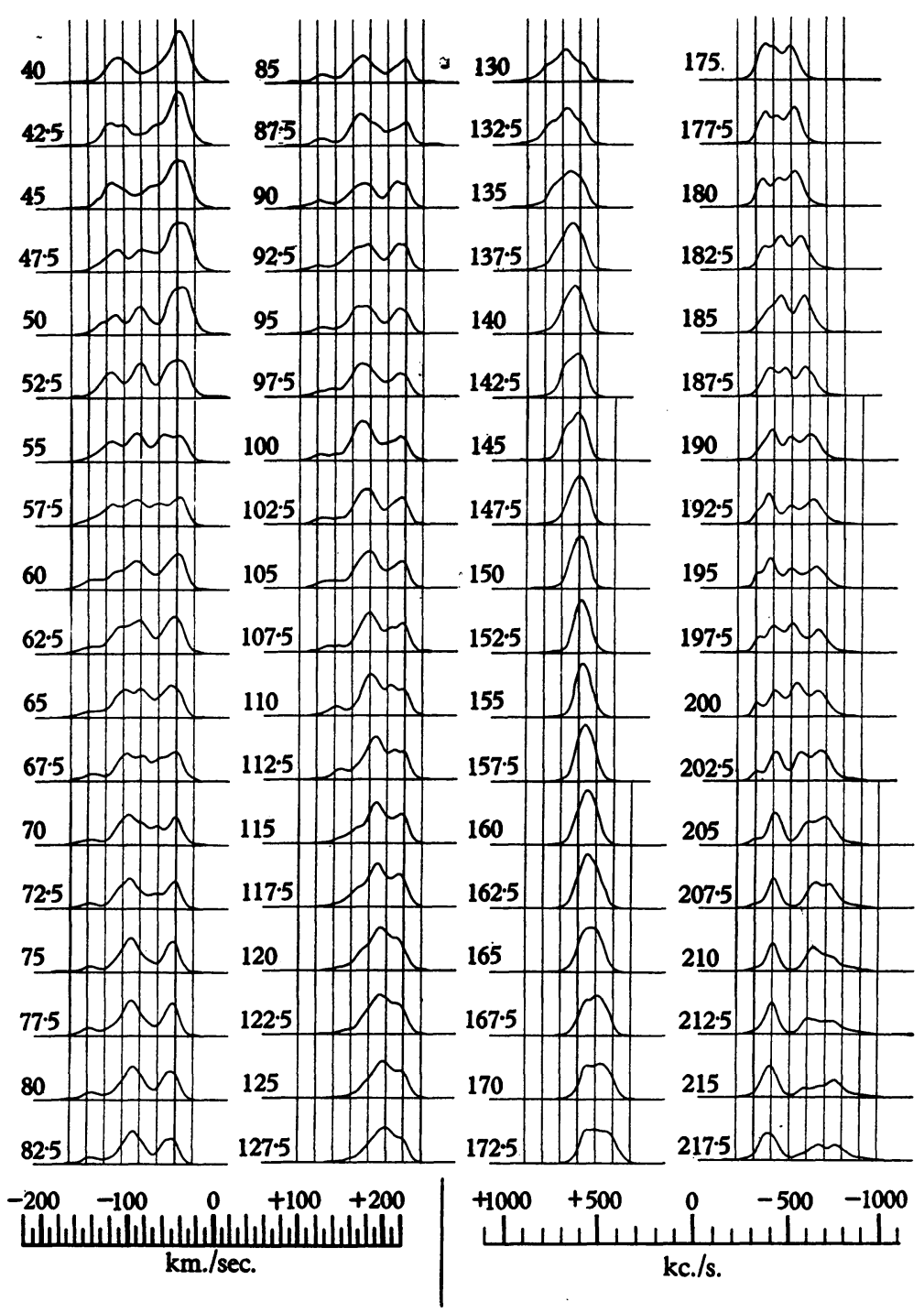

Fig. 2. Line profiles, in the galactic plane. From $l=321^{\circ}{ }^{\circ}$ to $38^{\circ} 4$, the latitudes are given in the figure, from $l=40^{\circ}$ to $l=217^{\circ} 5$, the latitude is $0^{\circ}$. The vertical lines are at $20 \mathrm{~km}$. $/ \mathrm{sec}$. intervals.

do not have a striking excess of $2 \mathrm{I}-\mathrm{cm}$. emission. Most of the gas in the very dense clouds may be molecular.

(8) E. Raimond studied two regions of $10^{\circ} \times 10^{\circ}$ each, centred around the $\zeta$ Persei association $\left(l=129^{\circ}, b=-15^{\circ}\right)$ and the Lacerta aggregate $\left(l=67^{\circ}, b=-14^{\circ}\right)$, which have been investigated optically by Blaauw [3] and Blaauw and Morgan [4]. 
No conspicuous features were found in the $\zeta$ Persei region, although there is a slight indication that the line of maximum intensity bulges out towards the association in a velocity-longitude plot.

In the Lacerta region an outstanding maximum shows up between $l=62^{\circ} \cdot 5$ and $70^{\circ}, b=-12^{\circ} 5$ and $-20^{\circ}$. It appears both in the line profiles and in a plot of optical depth, corrected for cloud velocities, against velocity and latitude. The relative velocity of the centre of this mass $\left(l=67^{\circ}, b=-16^{\circ}\right)$ with respect to the aggregate is $-17 \mathrm{~km}$. $/ \mathrm{sec}$. At some

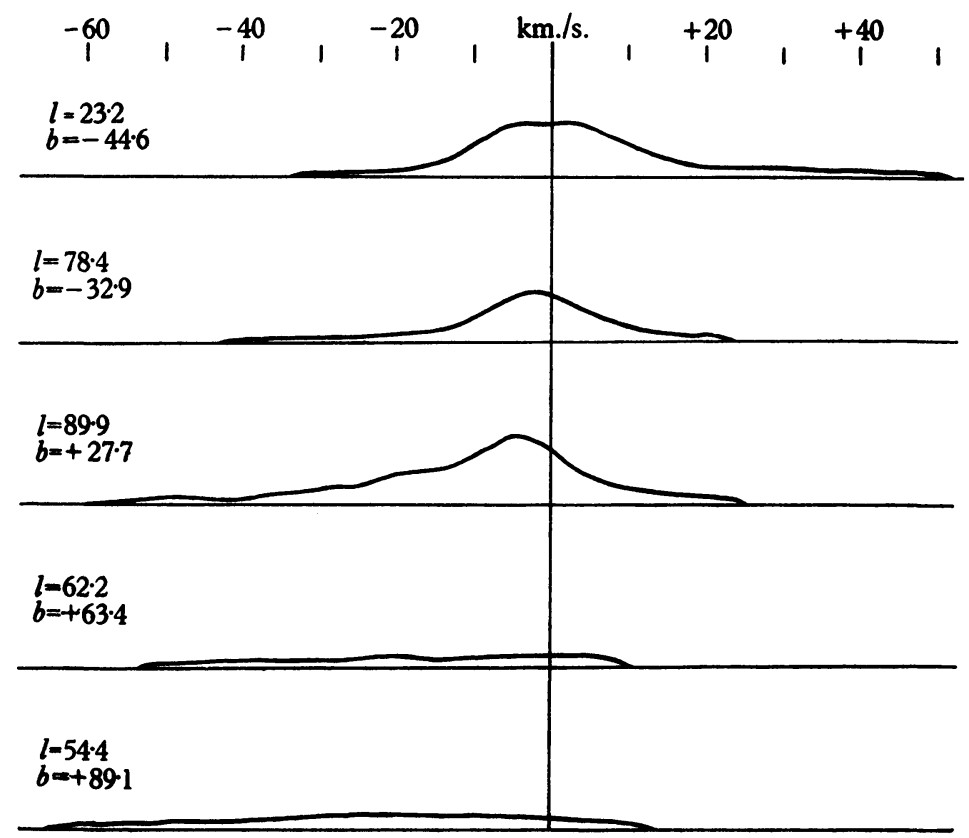

Fig. 3. Sample line profiles at high galactic latitudes. The middle profile is at the celestial North Pole, the bottom profile at the galactic North Pole.

points, there are clearly visible bulges of the lines of equal optical depth, away from the aggregate. If the dimensions of this hydrogen region are taken as $65 \times 65 \times 65$ parsecs, at a distance of 500 parsecs, we find a mean density $\bar{n}_{H}=\mathrm{r}$ atom $/ \mathrm{cm} .{ }^{3}$ and a total mass of the order of 5000 solar masses. Photographs show hardly any indications of dark clouds in this region.

(9) A first general impression of the hydrogen distribution at high latitudes was obtained from thirty line profiles made at latitudes $|b|>35^{\circ}$, evenly distributed over the visible part of the sky.

Most of these profiles show long wings of very low intensity (Fig. 3). Twelve have a clearly outspoken top at about the zero frequency, with 
maximum temperatures between $10^{\circ} \mathrm{K}$. and $26^{\circ} \mathrm{K}$. The remainder, although usually reaching maximum intensity at the zero frequency, has the appearance of a very broad low-intensity spectrum $\left(T_{\max } \leqslant 9^{\circ} \mathrm{K}\right.$.).

There appears to be a tendency for the long wings to extend farther to the negative velocity side. The mean maximum velocities are $-42 \mathrm{~km}$. $/ \mathrm{sec}$. and $+24 \mathrm{~km}$. $/ \mathrm{sec}$. There is no definite correlation with position.

Integration of the eighteen profiles with $T_{\max }<9^{\circ} \mathrm{K}$. shows that the mean total number of hydrogen atoms in a column of $1 \mathrm{~cm} .{ }^{2}$ cross-section is $\bar{N}_{H}=3 \times 10^{20} \mathrm{~cm} .^{-2}$. Assuming a length of 1000 parsecs for the line of sight we find an average density $\bar{n}_{H}=0.1 \mathrm{~cm} .^{-3}$. Separating the positive and negative velocities, we find that $70 \%$ is moving towards us.

From these first, very preliminary results, it may be concluded that a continuous stream of atomic hydrogen is moving towards the galactic plane. To attain the observed velocities, it must come from considerable heights above the plane. Some of the hydrogen may have reached these heights in high-velocity clouds, which have dispersed on their way up.

(10) From measurements of the maximum intensity of line profiles close to the horizon, L. Woltjer and G. Westerhout made an estimate of the extinction at $21 \mathrm{~cm}$. The transmission coefficient at the zenith was found to be $p=0 \cdot 9915 \pm \cdot 0008$, corresponding to an extinction of

$$
0.037 \mathrm{db} . \pm 0.003 \text {. }
$$

Expressed in magnitudes this gives an extinction coefficient

$$
\mathrm{k}=0^{\cdot m} 009 \pm \cdot 00 \mathrm{r} \text {. }
$$

This figure is in good agreement with data given by Van Vleck [5].

All profiles reduced since the publication of B.A.N. no. $45^{2}$ (1954) are corrected for the effect of extinction, and for the effect of the continuous radiation from the air and the ground, which influences the automatic volume control system of the receiver.

The work described above has been published in B.A.N. no. $475^{[6]}$.

The observations were made possible through the support of the Netherlands Organization for Pure Research (Z.W.O.)

The $2 \mathrm{I}-\mathrm{cm}$. equipment of the Netherlands Foundation for Radio Astronomy has recently been moved from the Kootwijk transmitting station to the Radio Astronomical Observatory, Dwingeloo. We are greatly indebted to the Netherlands Post and Telegraph service, who rendered us hospitality for so many years. 
The new, azimuthally mounted 25-metre paraboloid reflector at the Dwingeloo Observatory is approaching completion; two additional, equatorially mounted Würzburgs are also being installed.

It is a pleasure for me to express the thanks of all of us in the $2 \mathrm{I}-\mathrm{cm}$. research group to Ir G. A. Muller, whose fine engineering work-almost wholly a one-man job-has made it possible to obtain the exciting results reported here.

\section{REFERENCES}

[1] Kwee, K. K., Muller, G. A. and Westerhout, G. B.A.N. 12, 2 I I, no. 458, 1954 . [2] van de Hulst, H. C., Muller, C. A. and Oort, J. H. B.A.N. 12, 1 I 7, no. 452, 1954 .

[3] Blaauw, A. B.A.N. 11, 405, no. 433, 1952.

[4] Blaauw, A. and Morgan, W. W. Ap. J. 117, 256, 1953.

[5] van Vleck, J. H. M.I.T. Radiation Lab. Series, no. 13 (S. Silver, ed.), p. 64I, 195 I.

[6] Muller, C. A., Westerhout, G., Schmidt, M., Raimond, E., van de Hulst, H. C. and Ollongren, A. B.A.N. 13, 151 , no. 475, 1957.

\section{Discussion}

Greenstein: Optical observations by G. Münch, in unpublished investigations, show that most of the discrete clouds in front of stars 1000 parsecs from the galactic plane give systematically negative velocities from interstellar $\mathrm{K}$. This is an unexpected observation, because it had been thought that clouds at great heights above the galactic plane should be in the process of expulsion from the plane by high-temperature expansion processes. The importance of study of the high-velocity and high-velocity-dispersion $2 \mathrm{I}-\mathrm{cm}$. gas at considerable heights above the galactic plane cannot be overstressed. 